University of Wollongong

Research Online

Faculty of Engineering - Papers (Archive)

Faculty of Engineering and Information

Sciences

$1-1-2012$

\title{
Application of main flow data in the determination of boundary shear stress in smooth closed ducts
}

Yu Han

University of Wollongong, yh916@uow.edu.au

Shu-Qing Yang

University of Wollongong, shuqing@uow.edu.au

Nadeesha Dharmasiri

University Of Wollongong

Follow this and additional works at: https://ro.uow.edu.au/engpapers

Part of the Engineering Commons

https://ro.uow.edu.au/engpapers/5197

\section{Recommended Citation}

Han, Yu; Yang, Shu-Qing; and Dharmasiri, Nadeesha: Application of main flow data in the determination of boundary shear stress in smooth closed ducts 2012, 1175-1185.

https://ro.uow.edu.au/engpapers/5197

Research Online is the open access institutional repository for the University of Wollongong. For further information contact the UOW Library: research-pubs@uow.edu.au 


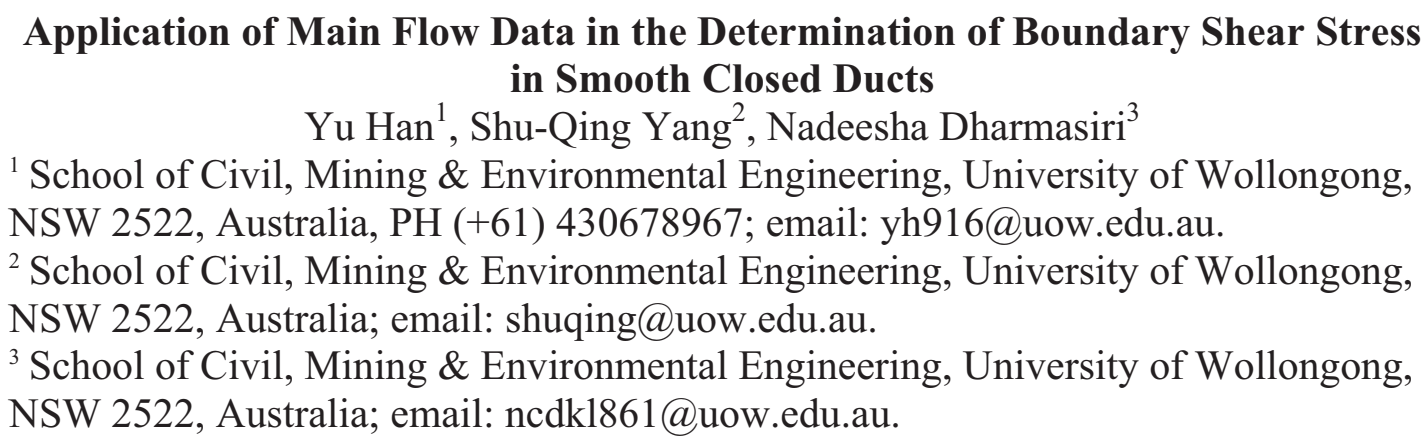

\begin{abstract}
Boundary shear stress plays an important role in momentum and mass transfers, but it is very difficult to determine experimentally. This paper develops a new method which can evaluate the boundary shear stress using the main flow data based on Momentum Balance Method (MBM). A theoretical relationship between the boundary shear stress and parameters of main flow region is established. The obtained results from MBM agree well with other methods, indicating that the new method is reasonable and reliable. The significant contribution of MBM is that the local boundary shear stress accurately and quickly can be determined by application of this new method. Hence, MBM may provide a useful tool for hydraulic engineers who need a simple and easy way to measure the boundary shear stress.
\end{abstract}

Subject Headings: Boundary shear; Reynolds stress, Secondary flow; Turbulent flow; Velocity distribution.

\title{
1 Introduction
}

Flow in a straight, rectangular closed duct has been attracted considerable attention from many researchers due to its simple boundary geometry and nonexistence of free surface effects. It is generally accepted that there are many similarities between flow in a rectangular open channel and closed duct [1-2]. Knowledge of the distribution of boundary shear stress around the wetted perimeter of an open channel or closed duct is a matter of fundamental importance in many hydraulic equations concerning resistance, sediment, dispersion, or cavitations' problems [3-4].

Most widely used method of skin friction measurements in turbulent boundary layer is the application of Preston tube[5]. It is understandable that Preston tube provides an easier way to operate in skin friction measurements, however, it also presents an obstruction to the flow. Some measurements of fluctuating boundary shear stress in wall-bounded flows have utilized heat transfer or mass transfer shear probes[6]. The main idea of operation of a heated-element probe is to know the rate of removal of heat by using a small metal film or wire. Hence, the instantaneous fluctuations of the wall shear stress can be measured when the probe is placed very near to or in contact with the wall. Using these intrusive probes, the difficulties are dominated by using these intrusive methods in reversing flows, vortices and highly turbulent flows. The hot-wire technique has also been used for the detailed investigation of wall shear stress [7]. However, the determination of the instantaneous skin-friction from hotwire measurements of the velocity gradient in the viscous sublayer is strongly 
affected by the proximity of the wall [8]. More recently, wall-shear-stress measurements were carried out using liquid-crystal coating, which is thin enough to detect the local wall-shear-stress distribution [9]. The near boundary region is the source of turbulent eddies, and it is also the place where turbulent energy is dissipated into thermal energy due to resistance [10]. Boundary region is the most difficult layer from which to obtain reliable experimental data. Thus, the accurate velocity data is very difficult to acquire relative to the data in the main flow region. The purpose of this paper is to present a theoretical relationship basis for boundary shear stress and main flow based on the momentum balance or the Momentum Balance Method (MBM), and this new method was verified using the experimental data, also the obtained results are compared with other methods, e.g. Log-law method (LLM), Reynolds shear stress method (RSM) and Viscous sub-layer Method (VLM). It is found that the agreement is reasonably acceptable. Thus this novel method can be developed to determine the boundary shear stress using the main flow data in order to avoid the viscous sub-layer measurements.

\section{Theoretical considerations}

Yang [11] established the momentum balance equation which is validated for a steady, uniform, and fully developed turbulent flow (see Figure 1). For any arbitrary shapes of channel or closed duct, taking the element "abcd" as an example, the gravitational force component for a unit length in the $x$-direction has to be balanced by the friction force on the interface of element "abcd" :
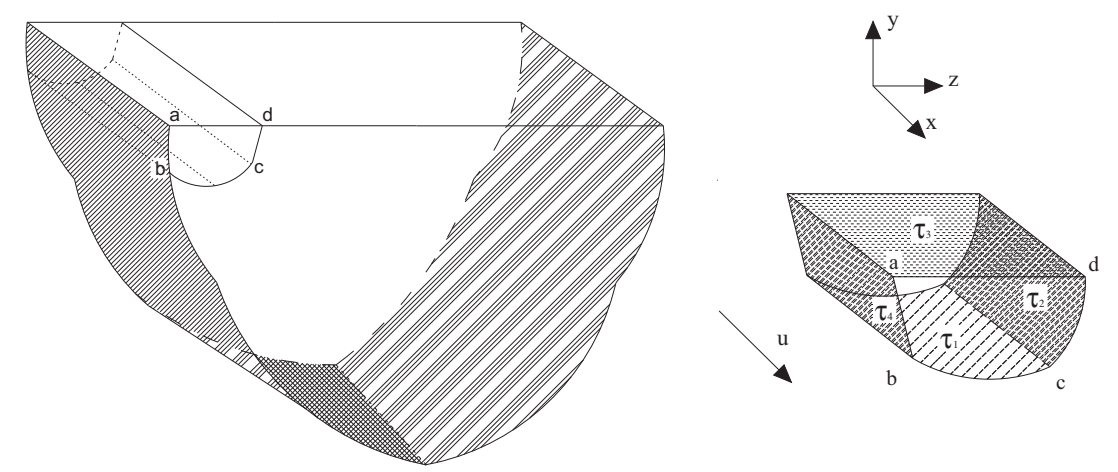

Figure 1. The calculation grids for arbitrary cross-sectional channel and coordinate system

$\rho g A_{a b c d} S_{f}=\oint_{s} \tau d s=\int_{b}^{c} \tau_{1} d s+\int_{c}^{d} \tau_{2} d s+\int_{d}^{a} \tau_{3} d s+\int_{a}^{b} \tau_{4} d s$

where $\rho=$ density of fluid; $g=$ gravitational acceleration; $A_{\text {abcd }}=$ area of flow region "abcd" shown in Fig. 1; $S_{f}=$ the energy slope; $\tau=$ the shear stress on the interface of "abcd" ; $s=$ the length of the interface, e.g. "bc", "cd" and "da" in Fig. $1 ; \tau_{1}, \tau_{2}$, $\tau_{3}=$ shear stresses on the interface "bc", "cd" and "da" respectively; and $\tau_{4}=$ the boundary shear stress to be determined. In streamwise direction, $\rho g A_{a b c d} S_{f}$ denote 
the gravitational force component for a unit length. The shear stress on the interface can be expressed as follows [12]:

$$
\tau_{x n}=\mu \frac{\partial u}{\partial \mathrm{n}}-\rho u v_{\mathrm{n}}-\rho \overline{u^{\prime} v_{\mathrm{n}}^{\prime}}
$$

where $\rho u v_{\mathrm{n}}=$ the momentum flux caused by mean flow ("n" represents the normal direction of the interface); and $v_{\mathbf{n}}=$ the velocity component of secondary flow normal to the interface. In the Cartesian coordinate system, Eq. 2 can be rewritten in the following form [13]:

$$
\begin{aligned}
& \tau_{x y}=\mu \frac{\partial u}{\partial y}-\rho u v-\rho \overline{u^{\prime} v^{\prime}} \\
& \tau_{x z}=\mu \frac{\partial u}{\partial z}-\rho u w-\rho \overline{u^{\prime} w^{\prime}}
\end{aligned}
$$

where $\tau_{x y}, \tau_{x z}=$ the shear stress on the horizontal and vertical interface, respectively; $u$ and $u^{\prime}, v$ and $v^{\prime}, w$ and $w^{\prime}$ are the mean and fluctuating velocities in $x, y$ and $z$ directions, respectively. In the main flow region, the viscosity effect can be ignored. Therefore, for an arbitrary control volume (see Figure 2 and Figure 3), the averaged sidewall shear stress $\bar{\tau}_{(y, y+\Delta y)}$ (acting on boundary from y to y+sy on the y-axis) can be computed and Eq. 1 can be written in the following form:

$$
\rho g A S=\oint_{s} \tau d s=\int_{0}^{z} \tau_{x y 1} d z+\int_{0}^{z} \tau_{x y 2} d z+\int_{y}^{y+\Delta y} \tau_{x z} d y+\int_{y}^{y+\Delta y} \tau_{(y, y+\Delta y)} d y
$$

where $A=$ the shadowed area (see Figure 2 and Figure 3); $\tau_{x y 1}, \tau_{x y 2}=$ the shear stress on the upper and lower horizontal interfaces, respectively; $\tau_{x z}=$ the shear stress on the vertical interface from the point $(z, y)$ to the point $(z, y+\Delta y)$. From experiments, the first three terms can be calculated from the measured 3D velocities, thus the sidewall averaged shear stress from $y$ to $y+\Delta y$ could be determined by using this momentum balance equation. Likewise, the bed shear stress can be evaluated from a vertical strip. Thus the wall shear stress can be calculated.

$$
\tau_{x z}(y+\Delta y / 2) \approx \frac{\int_{y}^{y+\Delta y} \tau_{x z}(y, y+\Delta y) d y}{\Delta y}
$$

When we started to calculation, one can set at the same strip but choosing different Momentum balance elements to build the equation, thus the averaged calculation value would be used. To check the MBM in application of determination of boundary shear stress, one must know the local friction velocity $u_{*}$ along the wetted perimeter with different methods, such as:

Log-law method (LLM): For a smooth boundary, the shear velocity $u_{*}$ can be determined from the measured mean velocity distribution $u(y)$ in conjunction with the logarithmic law.

$$
\frac{u}{u_{*}}=2.5 \ln \frac{u_{*} y}{v}+5.5
$$



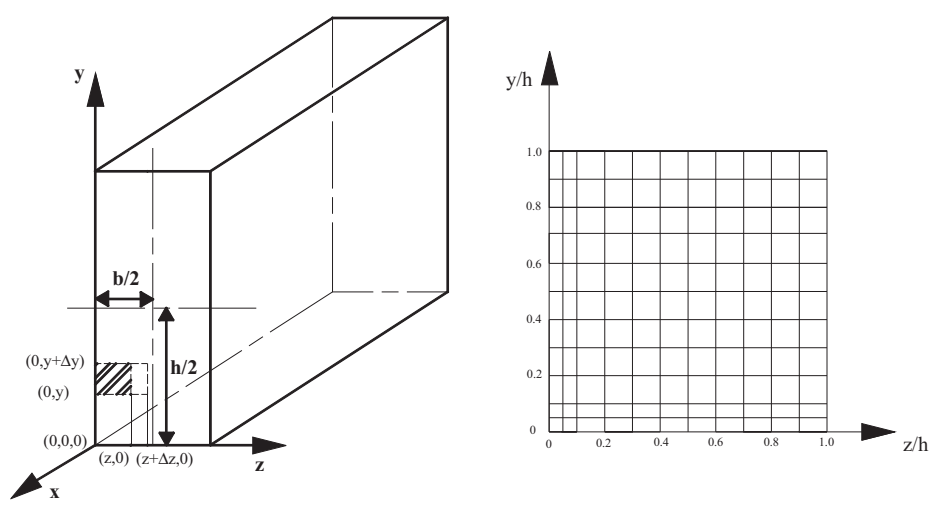

Figure 2. Orientation of test channel and calculation grids in measured crosssection by Tracy (1965)
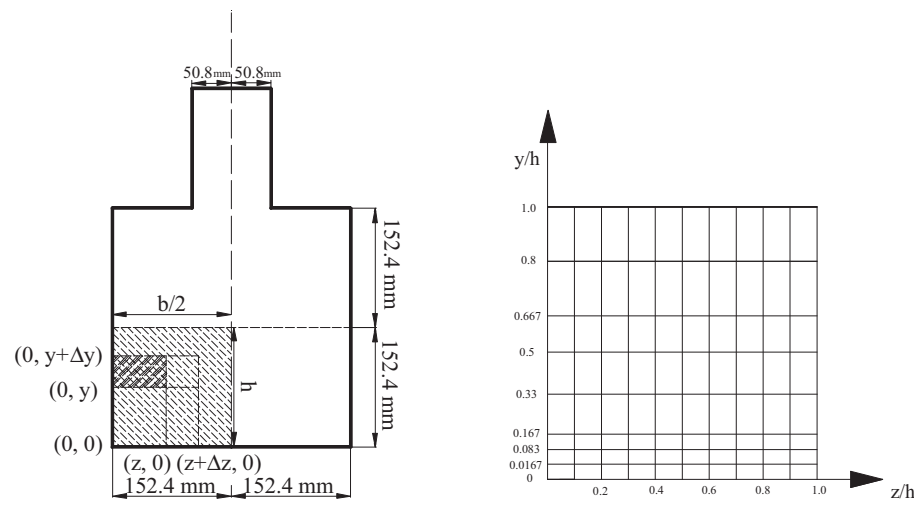

Figure 3. Orientation of test channel and calculation grids in measured crosssection by Tracy (1976)

where $v=$ kinematic viscosity. Eq. 6 is valid only in the inner region [14].

Reynolds shear stress method (RSM): $u_{*}$ can be determined from the measured Reynolds stress distribution[15]:

$\frac{\tau_{x y}}{\rho}=\overline{-u^{\prime} v^{\prime}}+v \frac{\partial u}{\partial y}=u_{*}^{2}\left(1-\frac{y}{h}\right)$

where $-\rho \overline{u^{\prime} v^{\prime}}=$ the Reynolds shear stress; $h=$ water depth. In near boundary region $y \approx 0$, the measured Reynolds shear stress is constant, thus the boundary shear stress is the Reynolds shear in the constant shear stress layer.

Viscous sub-layer Method (VLM): It is well known that the velocity in the viscous sublayer follows the linear distribution, i.e.,

$$
\frac{u}{u_{*}}=\frac{y u_{*}}{v}
$$

\section{MBM application to Smooth Closed Duct Flows}

In this section, two computational modules are comprised to prove the MBM conception, which are based on the experimental components carried out on a rectangular test duct and a complex boundary duct by Tracy [16-17] respectively. In these two test ducts, the air was supplied to a wind tunnel. In Tracy's (1965) experiment, the measurements were obtained by the rectangular closed duct $(\mathrm{b}=$ 
$127 \mathrm{~mm}, \mathrm{~h}=812 \mathrm{~mm})$. In the original paper the value of secondary currents are discernible only for the region of $0<2 y / b<1$ and $0<2 z / b<1$, so we will mainly focus on this domain. The bed shear stress distribution on the side wall and the bottom are in unity in this domain. Within Tracy's (1976) experiments regarding complex boundary, the flow characteristics could be analyzed by dividing the duct into two simple rectangular ducts as upper and lower parts. Experimental data [17] were only reported velocity measurements in lower part, hence, measurements in the compound channel only the lower part were used. If this lower duct was cut horizontal and vertically down its centre line, and then the flow in marked area would be the same as a rectangular duct. Thus for the rectangular duct illustrated (see Figure 3), the horizontal plane of symmetry, having zero shear stress, is analogous to the free surface, the calculation domain of these two ducts provide the physical model of a rectangular open channel. It is obvious the aspect ratios of these two models are equal to 1 . The parameters measured by Tracy [16-17] in calculation domains include $u, v, w, \overline{u^{\prime} v^{\prime}}$ and $\overline{u^{\prime} w^{\prime}}$, thus this dataset can be used for our purpose. In performing the computations, Tracy [16-17] datasets reported in literatures are used in the $11 \times 11$ (see Figure 2) and $10 \times 8$ (see Figure 3 ) mesh grid respectively. Studies on the distribution of the boundary shear stress were started to evaluate the friction velocity $\mathrm{u} *$ along the wetted perimeter in closed duct. Hence, LLM, VLM and RSM have been performed to investigate the value of $\mathrm{u} *$ along the wetted perimeter. As MBM outlined in theoretical considerations, the averaged local shear for each strips (see Figure 2 and Figure 3) would be further explored from Eq. 1 to 5 . It is noted each velocity profiles represented the velocity $u$ varied with the horizontal distance $z$ at a constant value of $\mathrm{y} / \mathrm{h}$, which can determine the $u_{*}$ results by Eq. 6 for each value of $y / h$, and the range are from 0.2 to 0.6 [16] and from 0.083 to 0.4 [17] respectively. Following this, the value of $\tau_{w}$ can be computed using $\rho u_{*}^{2}$ along the sidewall, thus, the obtained boundary shear stress using LLM is normalized by the mean wall shear stress, i.e., $\tau_{w} / \overline{\tau_{w}}$ and the data are presented in Figures 4 and 5 respectively.

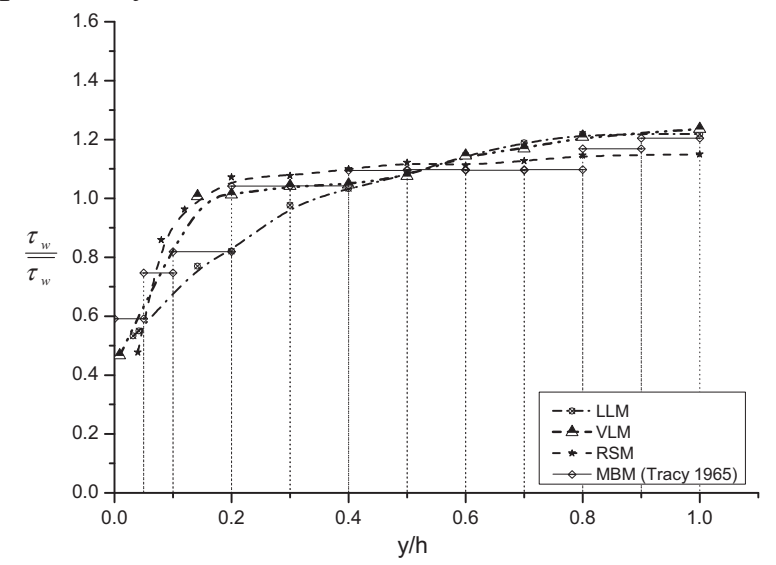

Figure 4. Comparison of averaged sidewall shear stress distributions between MBM and other three methods based on Tracy's (1965) data. 


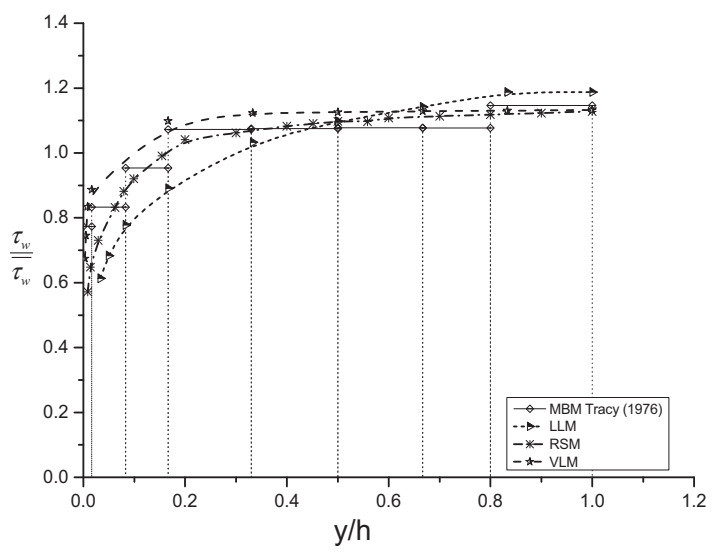

Figure 5. Comparison of averaged sidewall shear stress distributions between MBM and other three methods based on Tracy's (1976) data.

The velocity $u$ value closed to the wall $(z / h<0.02$ and $z / h<0.0167)$ would be used in calculation by $\operatorname{VLM}\left(\frac{u}{u_{*}}=\frac{z u_{*}}{v}\right)$ respectively. Here, it appears from VLM that the friction velocity $u_{*}$ would be computed, and define the boundary shear stress $\tau_{w}=\rho u_{*}^{2}$ along the duct perimeter (on the sidewall), then they obtained results are presented (see Figure 4 and Figure 5) as $\tau_{w} / \overline{\tau_{w}}$.

The measured the Reynolds shear stress near the boundary can be used to determine the boundary shear stress by RSM, i.e.:

$\overline{-u^{\prime} w^{\prime}}=u_{*}^{2}\left(1-\frac{z}{h}\right) \approx u_{*}^{2}$

This simplified methodology is also proposed by Nikora [18]. The distribution of $\overline{u^{\prime} w^{\prime}}$ relative to $u_{*}^{2}$ be shown from Tracy's paper [16-17], therefore, $-\rho \overline{u^{\prime} w^{\prime}}$ can be used to define wall shear stress, where all the measurements of $\overline{u^{\prime} w^{\prime}}$ closed to the wall $(\mathrm{z} / \mathrm{h}<0.02$ and $\mathrm{z} / \mathrm{h}<0.0167)$ were used in computation respectively. The data points (see Figure 4 and Figure 5) also show the variation of $\tau_{w} / \overline{\tau_{w}}$ along the duct perimeter made dimensionless which is computed by RSM.

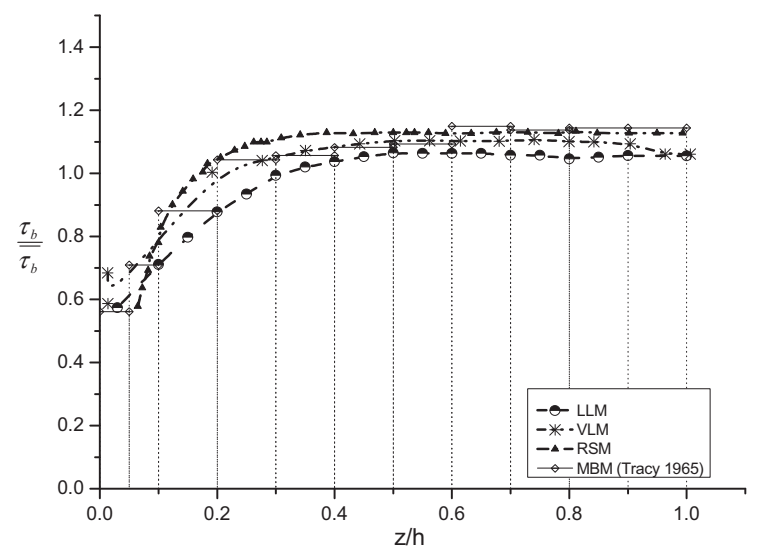

Figure 6. Comparison of averaged bed shear stress distributions between MBM and other three methods based on Tracy's (1965) data 


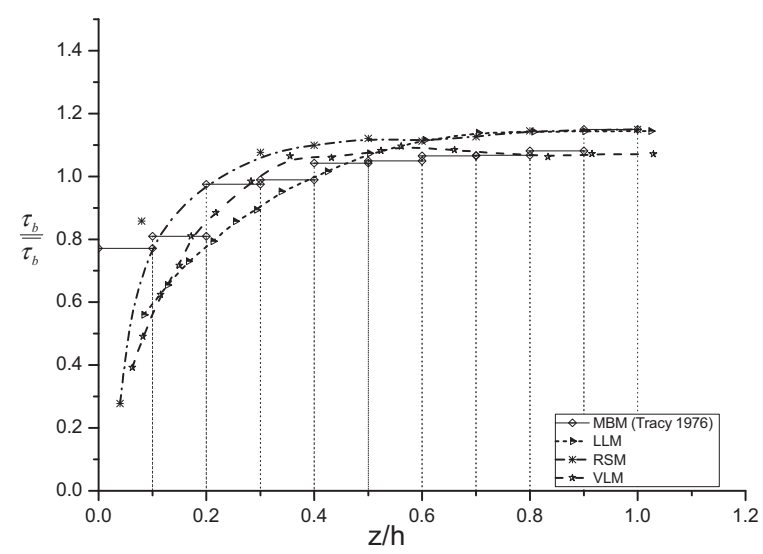

Figure 7. Comparison of averaged bed shear stress distributions between MBM and other three methods based on Tracy's (1976) data

Similarly, LLM, VLM and RSM are also applicable to assess the boundary shear stress distribution along the bed. Based on Tracy's measurement data in the boundary region, like velocity and Reynolds shear stress, one can determine the $u_{*}$ values for each $z / h$ using LLM and VLM. Following the calculation of $\tau_{b}=\rho u_{*}^{2}$, thus, the variation of bed shear stress $\tau_{b} / \overline{\tau_{b}}$ along duct perimeter are presented (see Figure 6 and Figure 7). As the distribution of $\overline{u^{\prime} v^{\prime}}$ relative to $u_{*}^{2}$ be showed in literature, it is used to investigate the boundary shear stress along the bed by RSM. The comparison shows that MBM works well and agrees with other methods. The bed shear stress rises from the corner and approaches stable value when it is nearly the symmetry plane.

Fig. 8 and Fig. 9 shows the comparison between the boundary shear stress obtained by MBM and other researchers' measured results, including Lawrence [19], Leutheusser [20] and Knight and Patel [1]. It is seen that the relative bed shear stress $\tau_{b} / \overline{\tau_{b}}$ of MBM agrees reasonably well with these mentioned data. These experiments were also conducted in the long rectangular ducts controlled by airflow through the system. In their experiments, values of local wall shear stress were deduced from the readings of Preston tubes, which were placed in a direct contact with the boundary. Experimental measurements for wall shear stress are made in a dimensionless form, i.e. $\tau_{b} / \overline{\tau_{b}}$ as well. It is seen that the relative bed shear stress $\tau_{b} / \overline{\tau_{b}}$ of MBM agrees reasonably well with these mentioned data. 


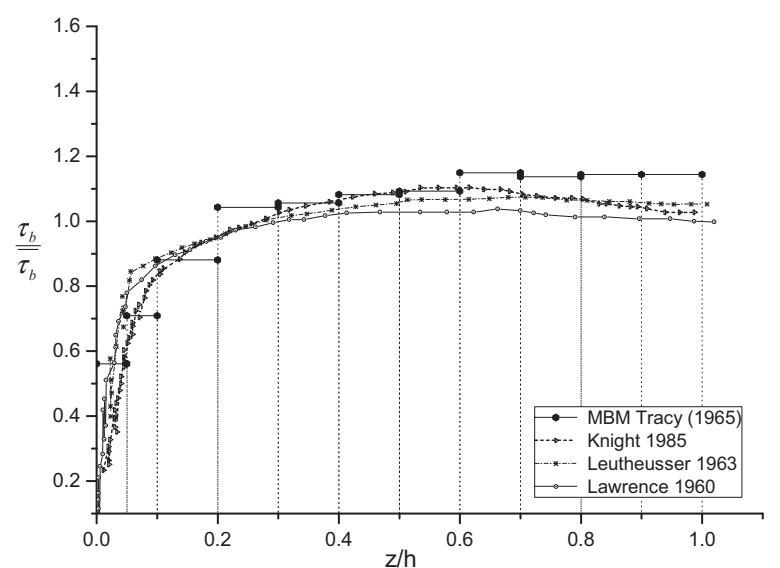

Figure 8. Comparison of averaged wallside shear stress distribution between MBM and other researcher's data for same aspect ratio

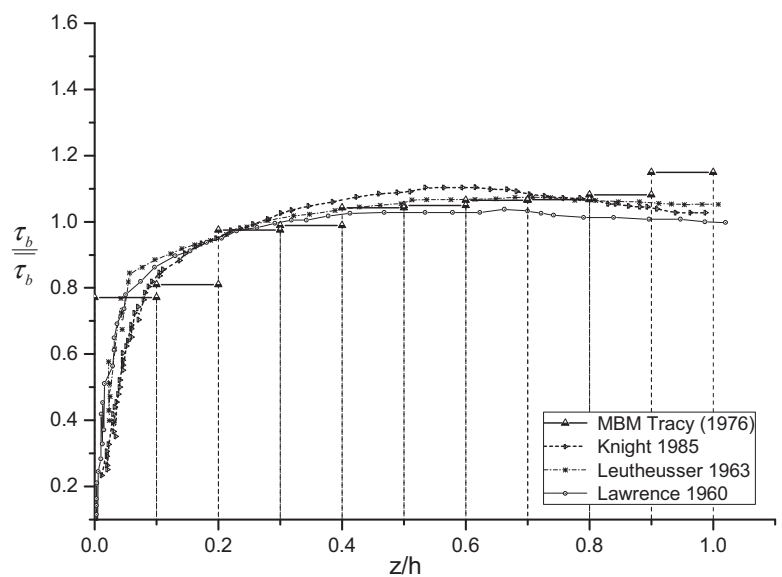

Figure 9. Comparison of averaged bed shear stress distribution between MBM and other researcher's data for same aspect ratio

To validate the accuracy of MBM, the relative error between MBM calculations (subscript $c$ ) and other methods (subscript $m$ ) has been defined as $E=\left|\tau_{c}-\tau_{m}\right| / \tau_{m}$. The sidewall shear stress has the averaged $E$ of $2.5 \%, 1.92 \%$ and $3.3 \%$ between MBM and LLM, VLM, RSM respectively (see Figure 4); The sidewall shear stress has the averaged $E$ of $-2.6 \%, 0.1 \%$ and $0.3 \%$ between MBM and LLM, VLM, RSM respectively (see Figure 5); In Figure 4, the maximum value of $E(=15.5 \%)$ occurs in the strip of 0.05-0.1. Tracy's two groups of experimental data prove MBM can provide a reasonably good estimation for the distribution of wall shear stress. Figure 6 shows the comparison along the bed by Tracy's 1965 dataset, it presents MBM works well and agrees with other methods (the averaged $E=-5.17 \%,-3.12 \%$ and $0.98 \%$ between MBM and LLM, VLM, RSM respectively), the maximum $E=13 \%$. Similarly, Figure 7 shows the calculation results from Tracy's 1976 data, the comparison shows that MBM works well and agrees with other methods (the averaged $E=-0.9 \%, 0.9 \%$ and $-0.3 \%$ between MBM and LLM, VLM, RSM respectively), the maximum $E=13.8 \%$ occurred in the corner region. The 
distribution of $\tau_{b} / \overline{\tau_{b}}$ is similar to the distribution of $\tau_{w} / \overline{\tau_{w}}$, so we only compared $\tau_{b} / \overline{\tau_{b}}$ with others' experimental data as presented (see Figure 8 and Figure 9 ), the maximum averaged $E$ occurred near its centre line. It should also be noted that the main flow values near the plane of symmetry used in the calculation are greater, hence a small error in measurements will result in significantly inaccurate. It should also be noticed that our model relied on Tracy's data, which calculates only certain layer due to the data limited, therefore, the grids cannot be detailing. The accuracy also depends on the main stream velocity on each grid points. Therefore, dividing the grids can lead to calculation accuracy. The present model simulates this behavior fairly well, and is actually in better agreement with present experiment data.

\section{4 . Conclusions}

A novel method (MBM) has been developed for determining boundary shear stress using main flow data based on the concept of momentum balance. The existing experimental data in smooth closed duct [16-17] have been well applied in MBM. In terms of the average wall or bed shear stress, comparison between three existing methods and other researcher's experimental data are shown MBM is possible to measure local boundary shear stress accurately and quickly under field conditions. The following conclusions can be drawn from this study:

1) MBM outlined the local boundary shear stress for turbulent flow over the wetted area can be conveniently analyzed in terms of the volume of flow towards a particular wetted boundary area. Thus, the main flow data that are important for calculation accuracy, on the basis of this concept, the flow cross-sectional area in different flow geometry can be divided and the grids used build momentum balance equation.

2) This study used smooth rectangular duct flows to test the MBM, and other existing methods were used for comparison including LLM, RSM and VLM. These comparisons showed that the MBM is comparable with these existing methods. When the estimated shear stress by MBM was compared with Lawrence (1960), Knight and Patel (1985) and Leutheusser (1963), it presents this method works well and agrees with these existing data. No empirical coefficient or assumption is involved in the method. This method's reliability mainly depends on the accuracy of velocity measurement in the main flow region.

3) For smooth rectangular duct flows, MBM can assess the distribution of boundary shear stress.Its performance is as good as other methods. The applicability of MBM could be extended to complex boundary conditions. The next step is to prove its applicability in complex flows. 
References:

[1] D. W. Knight and H. S. Patel, "Boundary Shear in Smooth Rectangular Ducts," Journal of Hydraulic Engineering, vol. 111, pp. 29-47, 1985.

[2] S. Y. Lim and S. Q. Yang, "Simplified model of tractive-force distribution in closed conduits," Journal of Hydraulic Engineering-Asce, vol. 131, pp. 322329, Apr 2005.

[3] S. Dey and M. F. Lambert, "Reynolds stress and bed shear in nonuniform unsteady open-channel flow," Journal of Hydraulic Engineering-Asce, vol. 131, pp. 610-614, Jul 2005.

[4] A. Dwivedi, et al., "Hydrodynamic Forces Generated on a Spherical Sediment Particle during Entrainment," Journal of Hydraulic Engineering, vol. 136, pp. 756-769, 2010.

[5] J. H. Preston, "The Determination of Turbulent Skin Friction by Means of Pitot Tubes," Journal of the Royal Aeronautical Society, vol. 58, pp. 109-121, 1954.

[6] A. Wietrzak and R. M. Lueptow, "Wall shear stress and velocity in a turbulent axisymmetrical boundary-layer," Journal of Fluid Mechanics, vol. 259, pp. 191-218, Jan 251994.

[7] H. H. Bruun, Hot-wire anemometry: principles and signal analysis: Oxford University Press, 1995.

[8] H. H. Fernholz, et al., "New developments and applications of skin-friction measuring techniques," Measurement Science and Technology, vol. 7, pp. 1396-1409, 1996.

[9] D. C. Reda, et al., "New methodology for the measurement of surface shear stress vector distributions," Aiaa Journal, vol. 35, pp. 608-614, Apr 1997.

[10] N. Chien, and Wan, Z., Mechanics of sediment transport: Reston, Virginia., 1999.

[11] S.-Q. Yang, "Depth-Averaged Shear Stress and Velocity in Open-Channel Flows," Journal of Hydraulic Engineering-Asce, vol. 136, pp. 952-958, Nov 2010.

[12] S. Q. Yang, "Conditionally averaged turbulent structures in 2D channel flow," Proceedings of the Institution of Civil Engineers-Water Management, vol. 163, pp. 79-88, Feb 2010.

[13] J. Guo, et al., "Modified log-wake law for zero-pressure-gradient turbulent boundary layers," Journal of Hydraulic Research, vol. 43, pp. 421-430, 2005 2005.

[14] I. Nezu, and Nakagawa, H., Ed., Turbulence in open-channel flows. Rotterdam, Netherlands: Balkema Publishers, 1993, p.`pp. Pages.

[15] I. Nezu and W. Rodi, "Open channel flow measurements with a laser doppler anemometer," Journal of Hydraulic Engineering-Asce, vol. 112, pp. 335-355, May 1986.

[16] H. J. Tracy, "Turbulent flow in a three-dimensional channel," J. Hydraul. Div.Am. Soc. Civ. Eng, vol. 91, pp. 9-35, 1965.

[17] H. J. Tracy, "The structure of a turbulent flow in a channel of complex shape," U.S. Geological Survey, Prof. Paper 983, 1976. 
[18] V. I. Nikora and D. G. Goring, "Fluctuations of Suspended Sediment Concentration and Turbulent Sediment Fluxes in an Open-Channel Flow," Journal of Hydraulic Engineering, vol. 128, pp. 214-224, 2002.

[19] L. C. Hoagland, "Fully developed turbulent flow in straight rectangular ductssecondary flow, its cause and effect on the primary flow (thesis). Technical Report No. 2," OOR-1935.2 United StatesTue Feb 05 15:11:00 EST 2008DTIE; NSA-15-005150English, 1960.

[20] H. J. Leutheusser, "Turbulent Flow in Rectangular Ducts," Journal of the Hydraulics Division, Proc. of ASCE, vol. 89, pp. 1-19, 1963. 